\title{
Laparoscopic inguinal Hernia Repair (Total Extra-Peritoneal): Tips and Tricks
}

Bhattarai SM, Simkhada G, Shah T, Gupta RK

GI and MIS unit, B.P. Koirala Institute Of Health Sciences, Dharan, Nepal.

Correspondence: Dr. S.M. Bhattarai, Junior Resident, B.P. Koirala Institute Of Health Sciences, Dharan, Nepal. Email: smbhattarai@gmail.com

\begin{abstract}
Introduction: Inguinal hernia is one of the major problems at B.P. Koirala Institute of Health Sciences (BPKIHS), a tertiary referral center. We perform Laparoscopic hernia repair as a routine procedure for groin hernias. This technique is highly demanding and complex. In our country the development of laparoscopic technique is still in a developing phase.
\end{abstract}

Aims and objective: To present technical tips and trick in LIHR, which we experienced, as lead to preventing intra/post operative complication.

Results: We have performed a total of 129 groin hernia repairs in 105 patients with $\mathrm{M}: \mathrm{F}=26: 1$ and mean age $41.97 \pm 20$ (16-85). The mean length of hospital stay was $1.14 \pm 0.35$ days. Eight patients were converted to TAPP and 1 converted to open hernia repair due to operative difficulty. The complications encountered are pain (20), seroma(8) and epididymitis(2).

Conclusion: In our experience LIHR (TEP) is safe method for routine management of groin hernias.

Key words: Groin hernia; LIHR; TEP 